\title{
Clinical and paraclinical aspects of HIV infected patients with first-line antiretroviral regimens
}

\author{
Ina Bîstrițchi ${ }^{1 *}$, Tiberiu Holban", Constantin Spânu ${ }^{2}$ \\ From The 9th Edition of the Scientific Days of the National Institute for Infectious Diseases Prof Dr Matei \\ Bals \\ Bucharest, Romania. 23-25 October 2013
}

\section{Background}

We aimed to assess the clinical, immunologic and virologic evolution of naive HIV-infected patients, who were treated with first-line regimens of highly active antiretroviral therapy (HAART) during 1 year.

\section{Methods}

The study included 149 adult patients diagnosed with HIV/AIDS infection (average age $36.28 \pm 0.81$ years), supervised in the specialized department of the Clinical Hospital of Infectious Diseases "Toma Ciorbă". The transmission of the HIV infection was in $87.25 \%$ (130) of the patients heterosexual and in $12.75 \%$ (19) by intravenous drug use. Out of the 149 patients who have initiated HAART, 94 (63.09\%) were detected late with a number of CD4<350 cells/cmm, out of which $56(37.58 \%)$ patients were detected very late with a number of $C D 4<200$ cells/ $\mathrm{cmm}$. At HAART initiation, undetectable HIV RNA had 17 (11.41\%) patients and HIV RNA $>100,000$ copies/mL had $46(30.87 \%)$ patients. Out of all patients who received HAART during the first 48 weeks, 53 (35.57\%) patients received AZT+3TC+EFV (regimen 1), 65 (43.62\%) patients - AZT+3TC+NVP (regimen 2) and 31 (20.81\%) patients - TDF+FTC+EFV (regimen 3). The late diagnosis is defined by the presence of AIDS associated diseases and/or the level of CD4<350 cells/cmm.

\section{Results}

More than half $(63.09 \%)$ of the investigated patients showed severe immunity suppression and/or clinical AIDS associated diseases. The most frequent AIDS related diseases were oropharyngeal candidiasis (55.03\%), tuberculosis $(22.82 \%)$, wasting syndrome (13.10\%), herpes zoster $(8.28 \%)$, Kaposi's sarcoma $(2.76 \%)$ and HIV encephalopathy (1.38\%). Prior to initiation of HAART, 73.83\% of the investigated patients were in AIDS stage (A3-10.07\%, B3-27.52\%, C2-6.71\% and C3-29.53\%). CD4 increase was for the first regimen from 189.54 \pm 13.25 to $290.39 \pm 21.13$ cells $/ \mathrm{cmm}$, representing an increase from baseline 1.53-fold ( $\mathrm{p}<0.001)$, for the second regimen - from $139.02 \pm 10.04$ to $272.55 \pm 19.11 \mathrm{cells} / \mathrm{cmm}$, an increase of 1.96-fold, which is a significant difference similar to the first regimen $(\mathrm{p}<0.001)$, and for the third regimen - from $163.42 \pm 22.4$ to $256.87 \pm 68.33$ cells $/ \mathrm{cmm}$, an increase of 1.57 -fold ( $>0.05$ ).

\section{Conclusion}

This study showed that more than half (63.09\%) of HIV/ AIDS - infected patients were detected late, with the number of T-lymphocytes $\mathrm{CD} 4<350$ cells $/ \mathrm{cmm}$, with or without AIDS related conditions. The therapeutic efficiency of HAART regimens in HIV infected patients was a success both immunologically and virologically, for all administered regimens, but an undetectable viral load and more sustainable increase of the CD4 count is higher (1.96 times) was determined for patients with second regimen $(\mathrm{AZT}+3 \mathrm{TC}+\mathrm{NVP})$.

\section{Authors' details}

${ }^{1}$ Department of Infectious Diseases, Tropical and Medical Parasitology, State Medical and Pharmaceutical University "Nicolae Testemițanu", Chişinău,

Republic of Moldova. ${ }^{2}$ National Public Health Center, Republic of Moldova.

* Correspondence: ina.bistritchi@yahoo.com

'Department of Infectious Diseases, Tropical and Medical Parasitology, State Medical and Pharmaceutical University "Nicolae Testemițanu", Chişinău,

Republic of Moldova

Full list of author information is available at the end of the article 
doi:10.1186/1471-2334-13-S1-P7

Cite this article as: Bîstrițchi et al:: Clinical and paraclinical aspects of

HIV infected patients with first-line antiretroviral regimens. BMC

Infectious Diseases 2013 13(Suppl 1):P7.

Submit your next manuscript to BioMed Central and take full advantage of:

- Convenient online submission

- Thorough peer review

- No space constraints or color figure charges

- Immediate publication on acceptance

- Inclusion in PubMed, CAS, Scopus and Google Scholar

- Research which is freely available for redistribution 